\title{
OPEN Distinct microbial community along the chronic oil pollution continuum of the Persian Gulf converge with oil spill accidents
}

\author{
Maryam Rezaei Somee ${ }^{1}$, Seyed Mohammad Mehdi Dastgheib², Mahmoud Shavandi², \\ Leila Ghanbari Maman ${ }^{3}$, Kaveh Kavousi ${ }^{3}$, Mohammad Ali Amoozegar ${ }^{1 \bowtie}$ \& \\ Maliheh Mehrshad ${ }^{4} \bowtie$
}

The Persian Gulf, hosting ca. $48 \%$ of the world's oil reserves, has been chronically exposed to natural oil seepage. Oil spill studies show a shift in microbial community composition in response to oil pollution; however, the influence of chronic oil exposure on the microbial community remains unknown. We performed genome-resolved comparative analyses of the water and sediment samples along Persian Gulf's pollution continuum (Strait of Hormuz, Asalouyeh, and Khark Island). Continuous exposure to trace amounts of pollution primed the intrinsic and rare marine oil-degrading microbes such as Oceanospirillales, Flavobacteriales, Alteromonadales, and Rhodobacterales to bloom in response to oil pollution in Asalouyeh and Khark samples. Comparative analysis of the Persian Gulf samples with 106 oil-polluted marine samples reveals that the hydrocarbon type, exposure time, and sediment depth are the main determinants of microbial response to pollution. High aliphatic content of the pollution enriched for Oceanospirillales, Alteromonadales, and Pseudomonadales whereas, Alteromonadales, Cellvibrionales, Flavobacteriales, and Rhodobacterales dominate polyaromatic polluted samples. In chronic exposure and oil spill events, the community composition converges towards higher dominance of oil-degrading constituents while promoting the division of labor for successful bioremediation.

\author{
Abbreviations \\ PG Persian Gulf \\ HC Hydrocarbon \\ PAHs Polyaromatic hydrocarbons
}

Exposure to oil and gas derivatives in marine ecosystems rich in oil reservoirs is inevitable due to natural seepage. Intensive industrial oil exploration and transit over the last century have further increased the risk of pollution in these ecosystems ${ }^{1}$. The Persian Gulf is a relatively shallow evaporative basin that hosts more than $48 \%$ of the world's oil reservoirs. The largest recorded oil spill in the Persian Gulf dates back to $1991^{2}$. However, this ecosystem has been chronically exposed to oil pollution through natural seepage, accidental oil derivatives release from transit tankers or refinery facilities, and discharge of oily wastes and heavy metals from offshore drilling sites.

The two major water inputs of the Persian Gulf include the Indian Ocean surface water entering through the Strait of Hormuz and Arvandrood river freshwater influx from the northwest side. These two streams shape the water circulation of the Persian Gulf in two branches, one along the Iranian coast and the other one along the Arabian coast. The retention time of this semi-enclosed water body is estimated to be around 2-5 years, where the northern part of the Gulf contains the oldest water ${ }^{3}$. The denser and more saline water partially exits the

\footnotetext{
${ }^{1}$ Extremophiles Laboratory, Department of Microbiology, School of Biology and Center of Excellence in Phylogeny of Living Organisms, College of Science, University of Tehran, Tehran, Iran. ${ }^{2}$ Biotechnology and Microbiology Research Group, Research Institute of Petroleum Industry, Tehran, Iran. ${ }^{3}$ Laboratory of Complex Biological Systems and Bioinformatics (CBB), Institute of Biochemistry and Biophysics, University of Tehran, Tehran, Iran. ${ }^{4}$ Department of Ecology and Genetics, Limnology and Science for Life Laboratory, Uppsala University, Uppsala, Sweden. ${ }^{\boxplus}$ email: amoozegar@ut.ac.ir; maliheh.mehrshad@ebc.uu.se
} 
basin through the southern part of the Strait of Hormuz in depth. Therefore, the limited water circulation in the Persian Gulf prolongs the residence time of pollutants in the basin ${ }^{3}$.

While representing low abundances in the pristine marine community, intrinsic oil-degrading taxa bloom in response to oil pollution and play a critical role in the bioremediation process ${ }^{4}$. Because of their vigilance in responding to pollution, they could be considered as microbial indicators of trace oil pollution ${ }^{5}$.

In oil spill accidents, the natural marine microbial community exposed to oil; responds with fluctuating composition as different oil components are gradually degraded ${ }^{6}$. As the input water is carried along the Gulf's water circulation in the Persian Gulf, it is exposed to varying types of oil derivatives in different locations. Sporadic cultivation efforts have isolated naphthalene degrading (Shewanella, Salegentibacter, Halomonas, Marinobacter, Oceanicola, Idiomarina, and Thalassospira $)^{7}$ and crude oil utilizing (Acinetobacter, Halomonas, Alcanivorax, Marinobacter, Microbacterium, and Rhodococcus ${ }^{8}$ ) bacteria from the Persian Gulf water and sediment samples. A single $16 \mathrm{~S}$ rRNA amplicon study of the Mangrove forest's sediment shows a community dominated by Alteromonadales (Marinobacter and Idiomarina) and Oceanospirillales (Alcanivorax and Halomonas) orders from Gammaproteobacteria and genera Meridianimaribacter and Muricauda from the family Flavobacteriaceae ${ }^{9}$. However, the impact of chronic exposure to oil derivatives and recurring pollutions on the microbiome of the Persian Gulf, their oil bioremediation capability, and recovery potential remains largely unknown.

In this study, we aim to (i) explore the dynamics of the input microbial community along the pollution continuum (a gradient from low to high oil contamination) and (ii) understand their metabolic capability for oil bioremediation. To do so, we performed genome-resolved comparative analyses of the Persian Gulf's water and sediment samples. Additionally, our study aims to compare the microbial community of the Persian Gulf with the publicly available metagenomes of oil-polluted marine water $(n=41)$ and sediment $(n=65)$ samples.

\section{Materials and methods}

Sampling site description, sample collection, and DNA extraction. Three locations in the Persian Gulf (abbreviated as PG) were selected for sampling based on their different level of exposure to oil pollution. The PG receives its major marine water input from the Indian Ocean through the Strait of Hormuz in spring (peaking around May-June), while the Arvandrood river delta in the northwest feeds the Gulf with freshwater input $^{10}$. Sampling locations were selected based on their level of exposure to the oil pollution and sampled in spring and summer 2018 (Supplementary Table S1, sheet 1). Sampling was done once at each location. Water and sediment samples from the Gulf's input water at the Strait of Hormuz (27.06112 N, 56.24636 E) were collected in May 2018. Along the water circulation current, water and sediment samples close to Asalouyeh county in Bushehr province $(27.31767 \mathrm{~N}, 52.32542 \mathrm{E})$ and Khark Island $(29.13194 \mathrm{~N}, 50.18105 \mathrm{E})$ were also collected in May and September 2018, respectively. Asalouyeh hosts a wide variety of natural gas and petrochemical industries, and it's surrounding water is exposed to aromatic compounds pollution ${ }^{11}$. Khark Island is the most critical oil transportation hub of the Persian Gulf, continuously exposed to oil pollution ${ }^{12}$.

Water and sediment samples were collected using a Niskin bottle and a grab sampling device KC Denmark (Van Veen Grab $2500 \mathrm{~cm}^{2}$ ), respectively. Salinity, $\mathrm{pH}$, dissolved oxygen (DO) concentration, conductivity, and temperature of each sample were measured by HQ40D Portable Multi Meter (HACH). DO concentration was measured in $\mathrm{mg} / \mathrm{l}$ unit and triplicate. The average value of these triplicate measurements is represented in Supplementary Table S1. For on site DO concentration measurement, we transferred $1 \mathrm{~L}$ of the samples collected from $5 \mathrm{~m}$ depth to the glass bottle and measured the DO concentration by inserting the probe in water.

Chlorophyll-a concentration of water samples was measured according to the protocol described elsewhere ${ }^{13}$. Total petroleum hydrocarbon (TPH) content of water and sediment samples was measured by GC-FID method ${ }^{14}$. The PAH concentration of water and sediment samples were determined by GC-MS (Agilent 5975C Series, EPA 8270C standard) and HPLC (Agilent 1200 Series, ISO 13877 standard) techniques, respectively ${ }^{15}$. Since the TPH content of the Khark sediment sample was higher than $1 \mu \mathrm{g} / \mathrm{g}$, the carbon distribution of this sample was also determined using the Simulated Distillation (GC-SimDis) method based on ASTM D2887 standard ${ }^{16}$. Other elements (e.g., Al, As, Ba, Be, Ca, Cl, Co, Cr, Cu, Fe, K, Li, Mg, Mn, Na, Ni, P, Pb, Rb, S, Sr, Ti, U and Zn) of each sample were also detected by ICP-MS (Agilent 4500 Series) (Supplementary Table S1, ionic content).

For each sampling point, $20 \mathrm{~L}$ of water samples were collected from $5 \mathrm{~m}$ depth. Samples were pre-filtered through $20 \mu \mathrm{m}$ (Albet DP5891150, Germany), and $5 \mu \mathrm{m}$ pore-size (Albet DP5895150, Germany) filters (15 cm in diameter). Biomass was finally concentrated on $0.22 \mu \mathrm{m}$ pore-size cellulose acetate filters (Sartorius 11107$142-N$, Germany) using a peristaltic pump. Sediment samples were collected using a grab sampling device. Water filters and sediment samples were stored on dry ice for transfer to the lab.

A standard phenol-chloroform protoco ${ }^{17}$ was used for extracting community DNA from the water samples. Extraction of DNA from sediment samples was carried out using DNeasy PowerMax Soil DNA Extraction Kit (QIAGEN 12988-10, Germany) according to the manufacturer's instruction. Extracted DNA samples were sequenced using Illumina Novaseq 6000 platform (PE150) (Novogene, Hong Kong).

Estimation of 16S rDNA abundance by qPCR. Haloferax volcanii (IBRC-M 10248) and Escherichia coli (IBRC-M 11074) were selected to draw the standard curve as representative of domain archaea and bacteria, respectively. Their genomic DNA was extracted ${ }^{18}$ and the $16 \mathrm{~S}$ rDNA was amplified using universal primers [21F (TCCGGTTGATCCYGCCGG $(\mathrm{Y}=\mathrm{C} / \mathrm{T})$ ] and 1492R (GGTTACCTTGTTACGACTT) for archaea and 27F (AGAGTTTGATCMTGGCTCAG) and 1492R (GGTTACCTTGTTACGACTT) for bacteria) ${ }^{19}$. The concentration of DNA was measured using Nanodrop (Thermo Nanodrop One/One-C Micro Volume Spectrophotometers). Copy number of double-strand DNA was estimated according to the formula: number of copies per $\mu \mathrm{l}=\left(\right.$ concentration of PCR product $\left.(\mu \mathrm{l}) \times 6.022 \times 10^{23}\right) /\left(\right.$ length of PCR product $\left.(\mathrm{bp}) \times 1 \times 10^{9} \times 650\right)$ in which, 650 is the molecular weight of one base pair in double-strand DNA and $6.022 \times 10^{23}$ is Avogadro num- 
ber. Domain-specific 16S rRNA primers named 338F (ACTCCTACGGGAGGCAGCAG), 533R (TTACCGCGG CTGCTGGCAC) ${ }^{20}$ and Parch519F (CAGCCGCCGCGGTAA), ARC915R (GTGCTCCCCCGCCAATTCCT) ${ }^{21}$ were selected to detect Bacteria and Archaea respectively. The qPCR was performed using the Power SYBR Green PCR Master Mix (BIOFACT, South Korea) in the MIC real-time PCR system (BioMolecular Systems, Australia). The unknown 16S rRNA copy number of each sample was calculated according to the standard curves ( $\mathrm{R}^{2}$ value was higher than $99.0 \%$ in both curves). The total content of prokaryotes in each sample was calculated using the sum of $16 \mathrm{~S}$ rDNA copies of bacteria and archaea.

Reference metagenome collection. For comparative analyses, publicly available metagenomes deposited to sequence read archive (SRA) of the GenBank were screened for the available metagenomic datasets originating from oil-polluted marine water and sediment samples. A total of 41 marine water and 65 marine sediment metagenomic datasets were collected. A detailed description of these metagenomes is shown in Supplementary Table S3. Water samples originated from Norway (Trondheimsfjord, $n=17$ ), Deepwater Horizon (Gulf of Mexico, $n=13$ ), the northern part of the Gulf of Mexico (dead zone, $n=6$ ), and Coal Oil Point of Santa Barbara $(n=5)$. Sediment samples originated from DWH Sediment (Barataria Bay, $n=45)$, Municipal Pensacola Beach (USA, $n=16$ ), and a hydrothermal vent in the Guaymas Basin (Gulf of California, $n=4$ ).

Ribosomal RNA classification. A subset of 5 million reads was separated from each dataset, and the reads affiliated to ribosomal RNA genes (16S/18S) were detected using SSU-ALIGN ${ }^{22}$. BLAST comparison of putative prokaryotic 16S rRNA sequences against the SILVA reference database (release 132SSUParc) and their taxonomic affiliation was assigned based on their closest hit if the read was $\geq 90$ bp at the similarity threshold of $\geq 90$.

Non-metric multidimensional scaling (NMDS) analysis of oil-polluted marine water and sediment metagenomic samples worldwide together with the PG samples was performed using the vegan package in Rstudio based on Bray-Curtis dissimilarity of the abundance of unassembled 16S rRNA reads of metagenomes (order-level). The Alpha diversity of samples was also measured using the vegan package in Rstudio based on the Shannon-Wiener index.

Sequence assembly, binning, and annotation. Paired-end reads of the PG sequenced datasets were interleaved using reformat.sh and quality trimmed by bbduck.sh scripts of BBMap toolkit ${ }^{23}$. All trimmed sequences of each dataset were assembled separately using MEGAHIT (k-mer list 49, 69, 89, 109, 129 and 149) ${ }^{24}$. Only contigs $\geq 1 \mathrm{~kb}$ were binned into metagenome assembled genomes (MAGs) based on their different mapping depth and tetranucleotide frequency, using MetaBat 2 software ${ }^{25}$. Contamination and completeness of each MAG were evaluated using CheckM and MAGs with completeness above $40 \%$ and contamination lower than 5\% were considered for further analysis ${ }^{26}$. The taxonomic affiliation of bins was assigned using GTDB-tk ${ }^{27}$. Putative genes were predicted using Prodigal ${ }^{28}$ and preliminarily annotated using Prokka in the metagenomics $\operatorname{mood}^{29}$. Predicted protein sequences of each MAG were further annotated using eggNOG-mapper ${ }^{30}$ and PfamScanner ${ }^{31}$.

\section{Results and discussion}

Persian Gulf water and sediment samples along the oil pollution continuum. Water and sediment samples were collected along the circulation current of the Persian Gulf from Hormuz Island [HW (SAMN12878178) and HS (SAMN12878113)), Asaluyeh area (AW (SAMN12878179) and AS (SAMN12878114)), and Khark Island (KhW (SAMN12878180) and KhS (SAMN12878115)] (Fig. 1). Physicochemical characteristics and Ionic content of the collected samples are presented in the Supplementary Table S1. The GC-FID analyses showed high TPH and polyaromatic hydrocarbon (PAH) concentrations in the Khark sediment (KhS) (Supplementary Table S2). The GC-SimDis analysis showed that $\mathrm{C}_{25}-\mathrm{C}_{38} \mathrm{HCs}$ were dominant in the KhS $(\sim 60 \%)$, followed by $>\mathrm{C}_{40} \mathrm{HCs}(\sim 14 \%)$ (Supplementary Fig. S1). Chrysene, fluoranthene, naphthalene, benzo(a)anthracene and phenanthrene were respectively the most abundant PAHs in KhS. This pollution could originate from oil spillage due to Island airstrikes during the imposed war (1980-1988), sub-sea pipeline failures, and discharge of oily wastewater or ballast water of oil tankers (ongoing for $\sim 50$ years) ${ }^{12}$. The TPH of other water and sediment samples was below the detection limit of our method $(<50 \mu \mathrm{g} / \mathrm{L}$ and $1 \mu \mathrm{g} / \mathrm{g}$, respectively). qPCR-mediated estimates showed an increase in the proportion of bacteria from HW to AW and KhW (87-96\%) for water and from AS to KhS and HS (74-99\%) for sediment samples. In comparison, the archaea represent a reverse trend reaching the highest proportion in AS ( 25\%) (Supplementary Fig. S2 and Supplementary Table S1).

Distinct prokaryotic community composition along the oil pollution continuum of the Persian Gulf. In pristine marine environments, the internal feedback mechanisms of the microbial communities facilitate keeping a steady "average" composition despite changes imposed by factors such as temperature, nutrient supply, and physical mixing ${ }^{32,33}$. However, in the Persian Gulf, the continuous exposure to oil pollution in water samples causes spatial patchiness and a shift in the microbial community composition resulting in distinct compositions along the pollution continuum. The HW microbial community, which is the input water to the Gulf, had a microbial community similar to what was reported for other marine ecosystems. The community is dominated by Synechococcales, SAR11, SAR86, Flavobacteriales, Actinomarinales, and Rhodobacterales. Along the pollution continuum, the community shifted towards a higher relative abundance of Gammaproteobacteria (from 13.5 to $48 \%$ ) and Bacteroidetes (from 8 to 16\%) representatives (Fig. 2A). The relative abundance of phyla Cyanobacteria, Actinobacteria, Marinimicrobia, and order Cytophagales of the phylum Bacteroidetes decreased; inversely, the relative abundance of phyla Proteobacteria, Epsilonbacteraeota, and Firmicutes as well as orders Flavobacteriales and Balneolales of the phylum Bacteroidetes consistently increased from HW to AW and KhW (Fig. 2A). The order Synechococcales negatively responds to oil pollution in marine surface water ${ }^{34}$. Decreased 




Figure 1. Geographical location of the sampling sites along the pollution continuum of the Persian Gulf. Hormuz sampling point is located close to the Persian Gulf's marine input water, Asalouyeh sampling point is mainly exposed to aromatic oil contaminants and Khark sampling point is exposed to pollution with different crude oil derivatives. Sampling locations are marked with red squares. Figure was plotted using the open-source maps of the "rnaturalearth" library in R.

abundance of Synechococcales along the oil pollution continuum from HW to AW and KhW complied with our chlorophyll-a measurements $(0.24,0.091$, and $0.013 \mu \mathrm{g} / \mathrm{L}$, respectively) (Supplementary Table S1). This decreased chlorophyll-a concentrations along the pollution continuum highlight the adverse impact of pollution on the Gulf's primary production. More empirical evidence is needed to unravel the impact of decreased Synechococcales abundance and its relation to oil pollution. While oil pollution of the KhW and AW samples was below our detection limit $(50 \mu \mathrm{g} / \mathrm{L})$, their dominant prokaryotic community was remarkably similar to other oil-polluted marine samples ${ }^{4}$. Oceanospirillales, Flavobacteriales, Alteromonadales and Rhodobacterales had the highest relative abundance in KhW and the prokaryotic community of AW was mainly comprised of Alteromonadales, SAR86, Flavobacteriales, Rhodobacterales, and Thermoplasmata (Fig. 2A).

In response to oil contamination (e.g., in the form of an oil spill), relative abundance of oil-degrading microbes increases (e.g., Oceanospirillales, Flavobacteriales, Alteromonadales, SAR86, and Rhodobacterales) ${ }^{35}$. Representatives of SAR86 clade contain cytochrome P450 and dioxygenase genes that are involved in degrading aliphatic 

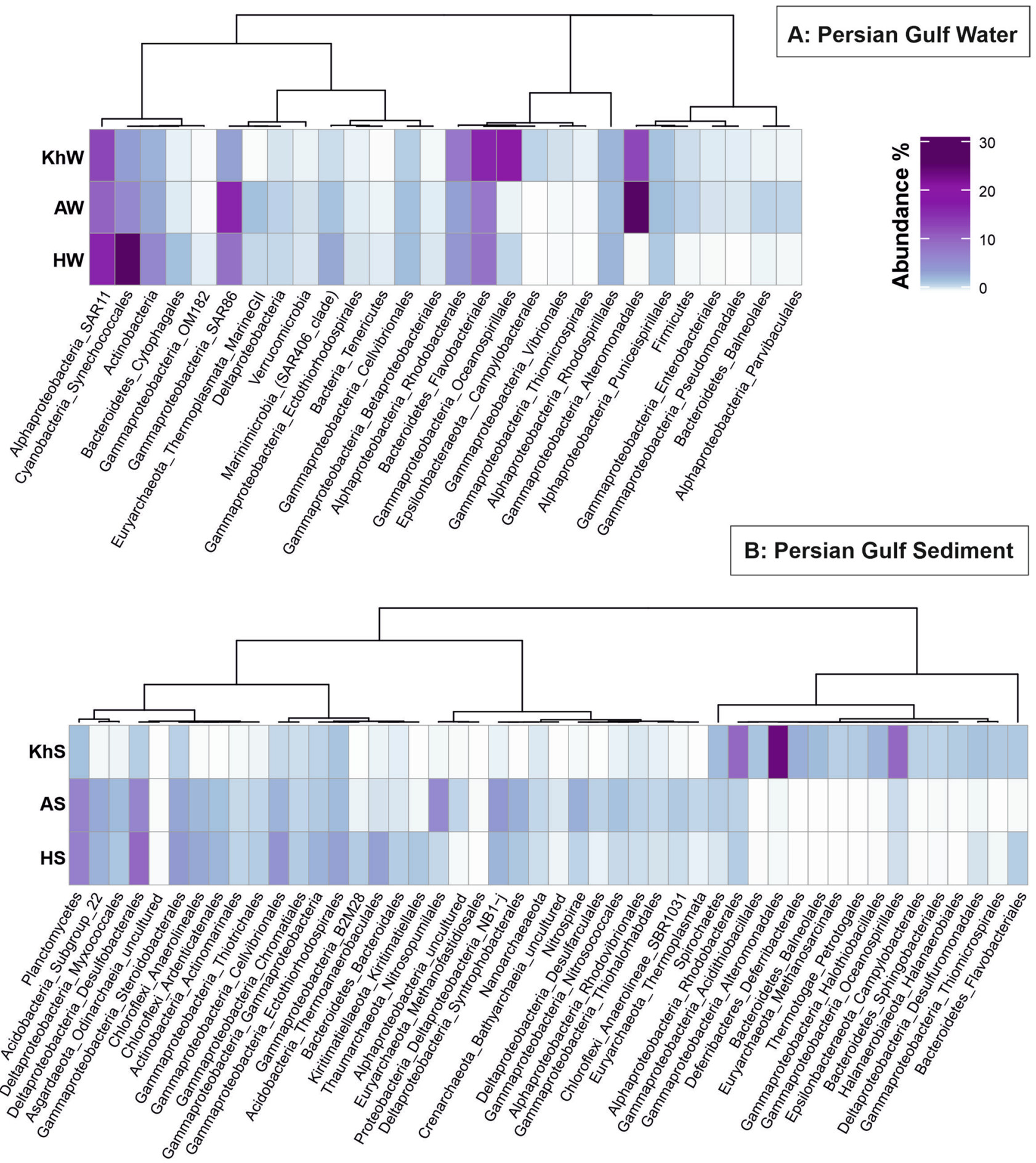

Figure 2. Prokaryotic community composition of the Persian Gulf water (A) and sediment (B) samples according to the abundance of $16 \mathrm{~S}$ rDNA reads in unassembled metagenomes. Column names are microbial taxa at the order level. For some taxa with lower frequency, the sum of orders is displayed in their corresponding higher taxonomic level. There are a total number of 28 and 48 taxa for water and sediment samples, respectively by which samples are compared. Rows are the name of samples. Dendrograms represent the clustering of columns based on Pearson correlation. Figure was plotted using "circlize" and "ComplexHeatmap" packages in R.

and aromatic xenobiotic compounds ${ }^{36}$. They reached the highest relative abundance in AW, where they were exposed to aromatic pollutants. Alteromonadales representatives encode enzymes for degrading recalcitrant and toxic branched-chain alkanes, and PAHs thus are mainly involved in the final steps of the degradation process ${ }^{5}$. 
They showed the highest relative abundance in AW, where most of the oil contaminants were low molecular weight aromatic compounds (Fig. 2A).

Oceanospirillales comprised ca. $20 \%$ of the KhW prokaryotic community (compared to ca. $1 \%$ in $\mathrm{HW}$ and AW), suggesting a recent recurring pollution. They prevail following marine oil pollution and are involved in the degradation of labile compounds such as non-branched alkanes and cycloalkanes ${ }^{37}$.

In sediment samples, KhS represented a distinct microbial profile from HS and AS. Alteromonadales, Rhodobacterales, Oceanospirillales, Deferribacterales, Halothiobacillales, and Balneolales (>2\%) representatives were enriched in KhS (Fig. 2B). The co-presence of the orders Methanosarcinals, Alteromanadales, and Thermotogae (Petrotogales) in the KhS hints at potential oil reservoir seepage around the sampling site since these taxa are expected to be present in oil reservoirs ${ }^{38}$. The main HC pollutants in the Asalouyeh are low molecular weight aromatic compounds that mainly influence the prokaryotic population in the water column and rarely precipitate into sediments hence the similarity of AS to HS microbial composition as they both experience low pollution rates.

Apart from oil-degrading Proteobacteria (e.g. Alteromonadales, Rhodobacterales, and Oceanospirillales), a diversity of sulfur/ammonia-oxidizing chemolithoautotrophic Proteobacteria were present in these sediments although at lower abundances e.g., (Acidithiobacillales (KhS 1.8\%), Chromatiales (HS 1.5, AS 1.1, KhS 0.85\%), Ectothiorhodospirales (HS 3.75, AS 2.3, KhS 1.7\%), Halothiobacillales (KhS 2.6\%), Thiotrichales (HS 1.5, AS 1.1, KhS 0.3\%), Thiohalorhabdales (HS 0.7, AS 1.2, KhS 0.5\%), Thiomicrospirales (KhS 1.5\%)) (Fig. 2B).

Sulfate-reducing bacteria (SRB) in HS comprised up to $16.2 \%$ of the community (Desulfobacterales, NB1-j, Myxococcales, Syntrophobacterales, and Thermodesulfovibrionia). Similar groups along with Desulfarculales, comprised the SRB functional guild of the AS ( 18.9\%). In comparison, Desulfuromonadales and Desulfobacterales were the SRB representatives in KhS with a total abundance of only $\sim 3.3 \%$. The lower phylogenetic diversity and community contribution of SRBs in KhS hint at the potential susceptibility of some SRBs to oil pollution or that HC degraders might outcompete them (e.g., Deferribacterales). Additionally, KhS was gravel-sized sediment (particles $\geq 4 \mathrm{~mm}$ diameter), whereas HS and AS samples were silt and sand-sized sediments ${ }^{39}$. The higher oxygen penetration in gravel particles of KhS hampers anaerobic metabolism of sulfate/nitrate-reducing bacteria hence their lower relative abundance in this sample (Fig. 2B).

Whereas in water samples, sulfur/ammonia-oxidizing chemolithoautotrophs such as Thiomicrospirales and sulfate/nitrate-reducing bacteria such as Desulfobacterales, NB1-j, Deferribacterales, Anaerolineales, Nitrosococcales, Nitrosopumilales, and Pirellulales were present in very small quantities (lower than $0.5 \%$ in each sample).

Chronic exposure to oil pollution shapes similar prokaryotic communities as oil spill events. We analyzed the prokaryotic community composition of 41 oil-polluted marine water metagenomes (different depths in the water column) from Norway (Trondheimsfjord), Deepwater Horizon (Gulf of Mexico), the northern part of the Gulf of Mexico (dead zone) and Coal Oil Point of Santa Barbara; together with 65 oil exposed marine sediment metagenomes (beach sand, surface sediments and deep-sea sediments) originating from DWH Sediment (Barataria Bay), Municipal Pensacola Beach (USA) and a hydrothermal vent in Guaymas Basin (Gulf of California) in comparison with the PG water and sediment samples (in total 112 datasets) (Supplementary Table S3). This extensive analysis allowed us to get a comparative overview of the impact of chronic oil pollution on the prokaryotic community composition.

Hydrocarbonoclastic bacteria affiliated to Oceanospirillales, Cellvibrionales (Porticoccaceae family), and Alteromonadales ${ }^{40}$ comprised a significant proportion of the prokaryotic community in samples with higher aliphatic compounds pollution e.g. DWHW.BD3 (sampled six days after the incubation of unpolluted water with Macondo oil), DWHW.he1, and DWHW.he2 (oil-polluted water samples incubated with hexadecane), DWHW.BM1, DWHW.BM2, DWHW.OV1 and DWHW.OV2 (sampled immediately after the oil spill in the Gulf of Mexico) (Fig. 3). Samples treated with Macondo oil, hexadecane, naphthalene, phenanthrene, and those taken immediately after the oil spill in the Gulf of Mexico had a significantly lower proportion of SAR11 due to the dominance of bloom formers and potential susceptibility of SAR11 to oil pollutants (Fig. 3).

Flavobacteriales and Rhodobacterales were present in relatively high abundance in almost all oil-polluted water samples except for those with recent pollution. Samples named NTW5, NTW6, NTW11, NTW12, which were incubated with MC252 oil for 32-64 days, represented similar prokaryotic composition dominating taxa that are reportedly involved in degrading recalcitrant compounds like PAHs in the middle-to-late stages of the oil degradation process (Alteromonadales, Cellvibrionales, Flavobacteriales, and Rhodobacterales). Whereas at the earlier contamination stages, samples represented a different community composition with a higher relative abundance of Oceanospirillales (e.g., NTW8, NTW9, NTW15, NTW16, and NTW17 sampled after 0-8 days incubation) (Fig. 3).

The non-metric multidimensional analysis of the prokaryotic community of 106 oil-polluted water and sediment samples, together with the PG samples, is represented in Fig. 4. Water and sediment samples expectedly represented distinct community compositions. The AW sample was placed near samples treated with phenanthrene and naphthalene in the NMDS plot showing the impact of aromatic compounds on its microbial community. The KhW sample was located near NTW13 in the plot, both of which had experienced recent oil pollution.

The orders Oceanospirillales, Alteromonadales, and Pseudomonadales were present in relatively high abundances in all oil-polluted water samples except for HW (PG input water) and samples collected from the northern Gulf of Mexico dead zone (GOMDZ) (Fig. 3). Persian Gulf was located in the proximity of the developing oxygen minimum zone (OMZ) of the Arabian Sea that is slowly expanding towards the Gulf of Oman ${ }^{41}$. Potential water exchange with OMZ areas could be the cause of higher similarity to the GOMDZ microbial community ${ }^{42}$.

Our results suggest that water samples with similar contaminants and exposure time to oil pollution enrich for similar phylogenetic diversity in their prokaryotic communities (Fig. 3). Marine prokaryotes represent vertical 


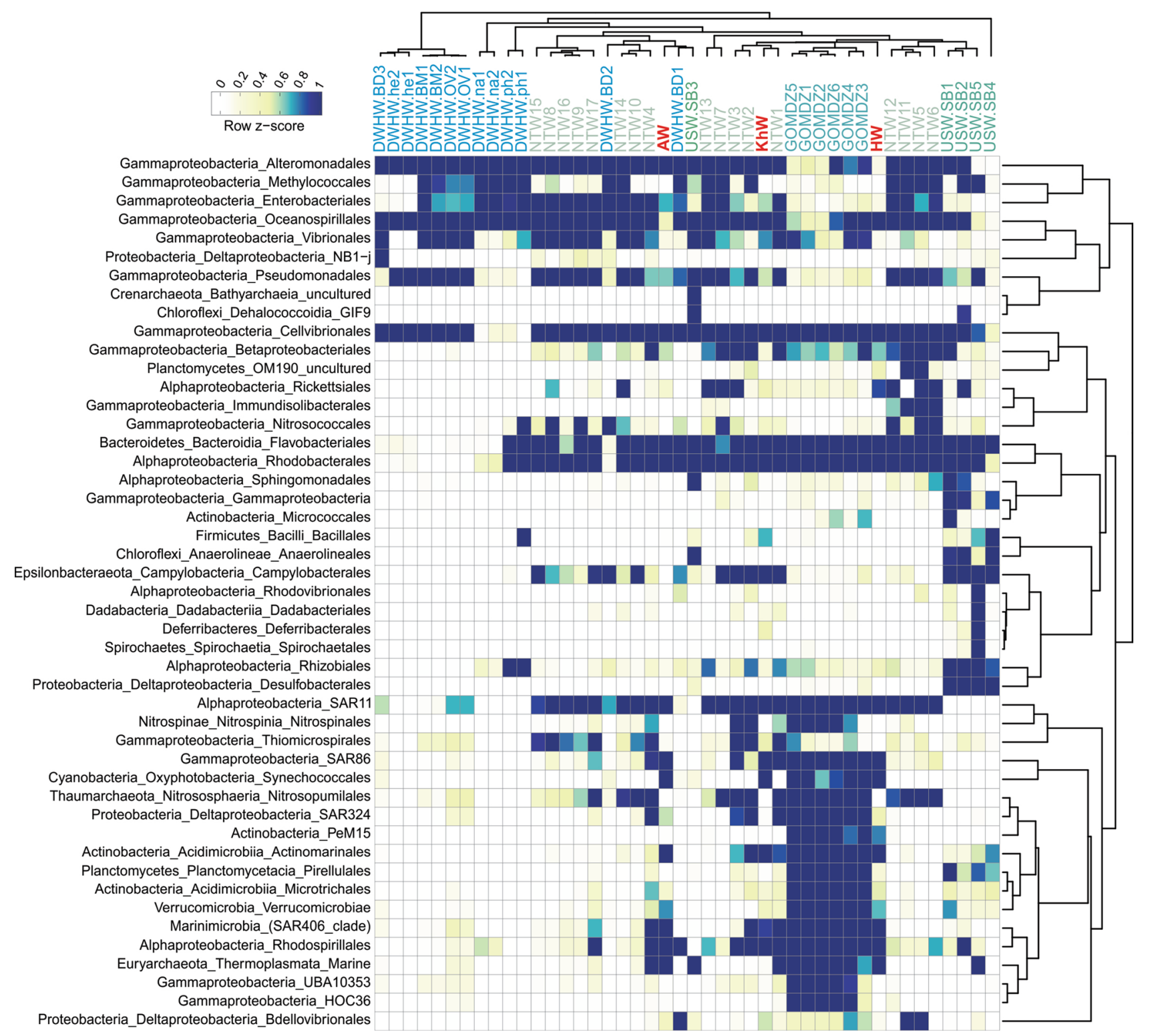

Figure 3. The abundance of unassembled $16 \mathrm{~S}$ rDNA reads from unassembled metagenomes of different oilpolluted water samples (41). Row names are microbial taxa at the order level. For taxa with lower frequency, the higher taxonomic level is shown (47 taxa in total). The right-hand dendrogram represents the clustering of rows based on the Pearson correlation. Columns are the name of water samples. Samples are clustered based on Pearson correlation and the color scale on the top left represents the row Z-score. Figure was plotted using "circlize" and "ComplexHeatmap" packages in R.

stratification with discrete community composition across the depth profile. According to our analyses, the prokaryotic communities of the oil-polluted areas are consistently dominated by similar taxa regardless of sampling depth or geographical location. We speculate that the high nutrient input due to crude oil intrusion into the water presumably disturbs this stratification and HC degrading microorganisms are recruited to the polluted sites where their populations flourish.

The inherent heterogeneity of the sediment prokaryotic communities is retained even after exposure to oil pollution, reflected in their higher alpha diversity (Supplementary Fig. S3). However, similar taxa dominate the community in response to oil pollution (Fig. 5).

In sediment samples, Deltaproteobacteria had the highest abundance, followed by Gammaproteobacteria representatives. Ectothiorhodospirales, Rhizobiales, Desulfobacterales, Myxococcales, and Betaproteobacteriales representatives were present in almost all samples at relatively high quantities (Fig. 5). Sulfate/nitrate-reducing bacteria were major HC degraders in sediment, showing substrate specificity for anaerobic HC degradation ${ }^{43}$. Desulfobacterales and Myxococcales were ubiquitous sulfate-reducers, present in almost all oil-polluted sediment samples ${ }^{44}$. Sulfate-reducing Deltaproteobacteria play a key role in anaerobic PAH degradation, especially in sediments containing recalcitrant $\mathrm{HC}$ types ${ }^{45}$. Members of Rhizobiales are involved in nitrogen fixation, 


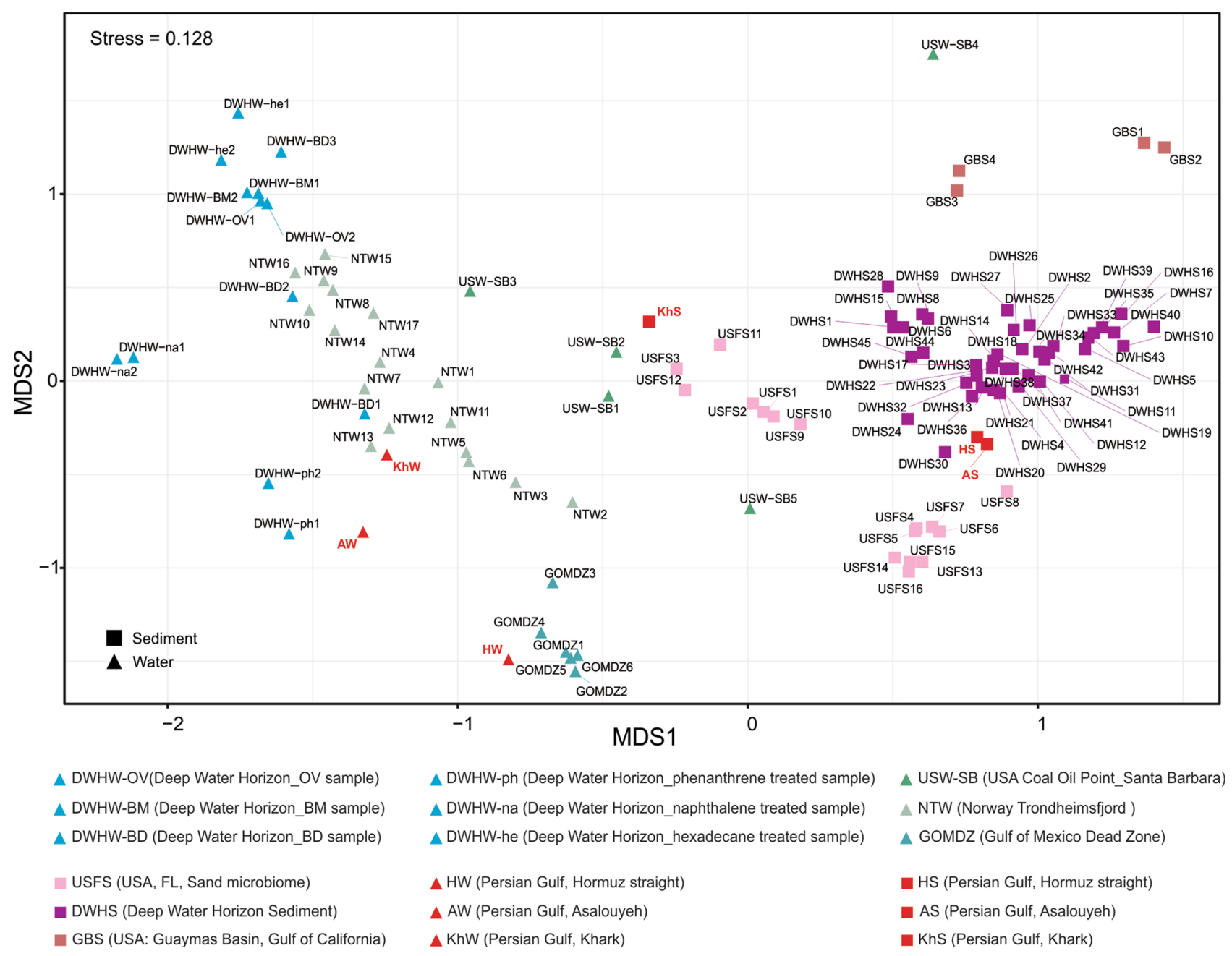

Figure 4. Non-metric multidimensional scaling (NMDS) of the Persian Gulf water and sediment metagenomes along with oil-polluted marine water and sediment metagenomes based on Bray-Curtis dissimilarity of the abundance of $16 \mathrm{~S}$ rDNA reads in unassembled metagenomes at the order level. Samples with different geographical locations are shown in different colors. PG water and sediment samples are shown in red. Water and sediment samples are displayed by triangle and square shapes, respectively. Figure was plotted using "vegan" library in $\mathrm{R}$.

which accelerates the HC removal process in the sediment samples ${ }^{46}$, and therefore their abundance increase in response to oil pollution (Fig. 5).

Prokaryotes involved in nitrogen/sulfur cycling of sediments are defined by factors such as trace element composition, temperature, pressure, and more importantly, depth and oxygen availability. In oil-polluted sediment samples, the simultaneous reduction of available oxygen with an accumulation of recalcitrant HCs along the depth profile complicates the organic matter removal. However, anaerobic sulfate-reducing HC degrading bacteria will cope with this complexity ${ }^{47}$. Prokaryotic communities of HS and AS samples represented similar phylogenetic diversity (Figs. 4,5). Their prokaryotic community involved in the nitrogen and sulfur cycling resembles the community of DWHS samples. The KhS sample had a similar prokaryotic community to deeper sediment samples collected from 30 to $40 \mathrm{~cm}$ depth (USFS3, USFS11, and USFS12) which could be due to our sampling method using a grab sampling device.

Our results show that the polluted sediments' sampling depth (surface or subsurface) defines the dominant microbial populations. Hydrocarbon degrading microbes had the ubiquitous distribution in almost all oil-polluted water and sediment samples including Oceanospirillales, Cellvibrionales, Alteromonadales, Flavobacteriales, Pseudomonadales, and Rhodobacterales. Mentioned orders along with Ectothiorhodospirales, Rhizobiales, Desulfobacterales, Myxococcales, and Betaproteobacteriales and also representatives of Deltaproteobacteria phylum dominated in sediment samples. However, their order of frequency varies depending on the type of oil pollution present at the sampling location and the exposure time.

Genome-resolved metabolic analysis of the Persian Gulf's prokaryotic community along the pollution continuum. A total of 82 metagenome-assembled genomes (MAGs) were reconstructed from six sequenced metagenomes of the PG (completeness $\geq 40 \%$ and contamination $\leq 5 \%$ ). Amongst them, eight MAGs 


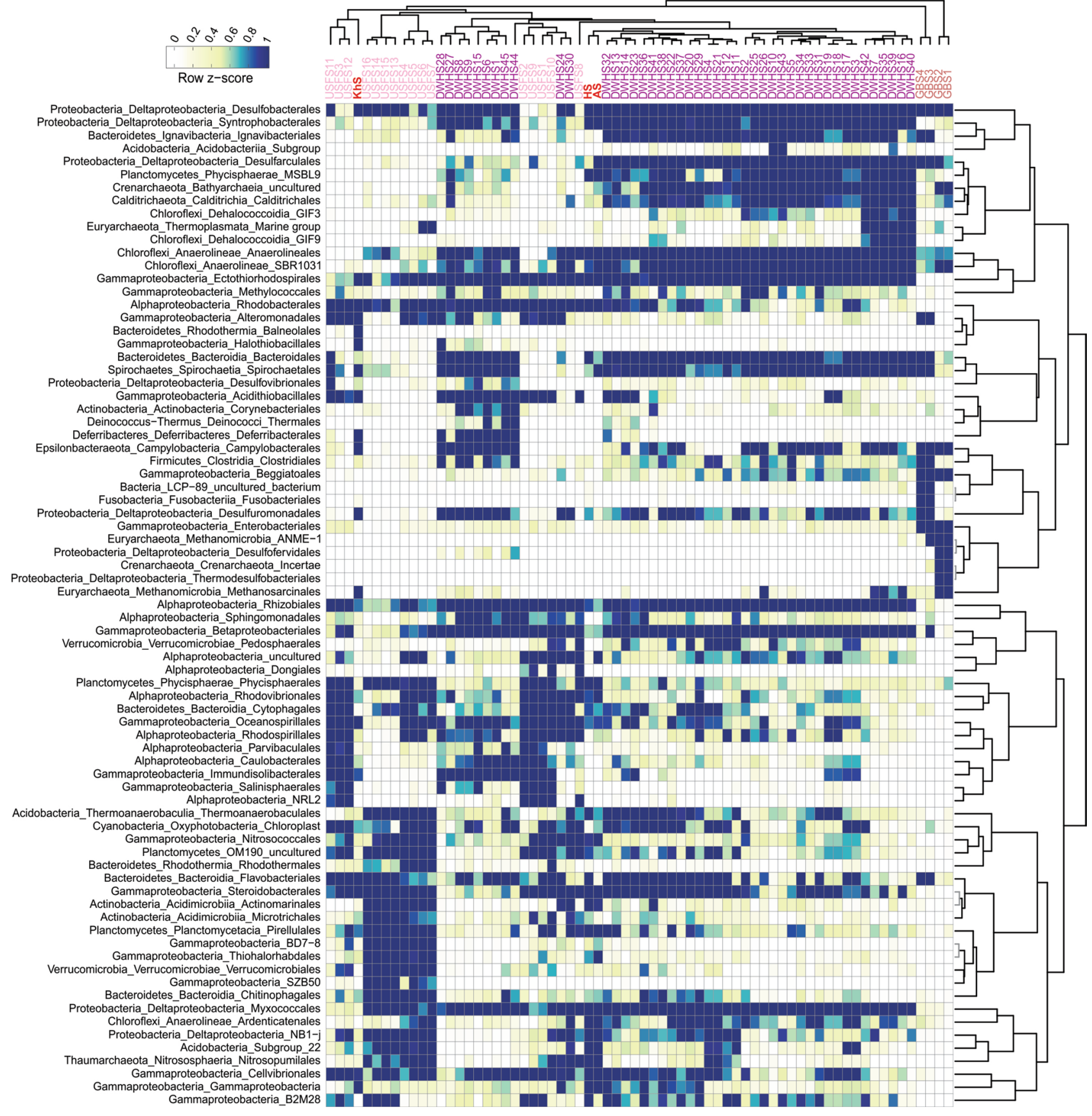

Figure 5. The abundance of unassembled $16 \mathrm{~S}$ rDNA reads from unassembled metagenomes of different oilpolluted sediment samples (65). Row names are microbial taxa at the order level. For taxa with lower frequency, the higher taxonomic level is shown (77 taxa in total). The right-hand dendrogram represents the clustering of rows based on the Pearson correlation. Columns are the name of sediment samples. Samples are clustered based on Pearson correlation and the color scale on the top left represents the row Z-score. Figure was plotted using "circlize" and "ComplexHeatmap" packages in R.

belonged to domain Archaea and 74 to domain bacteria. According to GTDB-tk assigned taxonomy (release89) (https://data.gtdb.ecogenomic.org/releases/release89/), reconstructed MAGs were affiliated to Gammaproteobacteria (36.6\%), Alphaproteobacteria (12.2\%), Flavobacteriaceae (9.7\%), Thermoplasmatota (5\%) together with some representatives of other phyla (MAG stats in Supplementary Table S4).

A collection of reported enzymes involved in the degradation of different aromatic and aliphatic HCs under both aerobic and anaerobic conditions was surveyed in the annotated MAGs of this study ${ }^{43,48-50}$. The KEGG orthologous accession numbers (KOs) of genes involved in $\mathrm{HC}$ degradation were collected, and the distribution of KEGG orthologues detected at least in one MAG $(n=76$ genes) is represented in Fig. 6.

A combination of different enzymes runs the oil degradation process. Mono- or dioxygenases are the main enzymes triggering the HC degradation process under aerobic conditions. Under anaerobic conditions, 


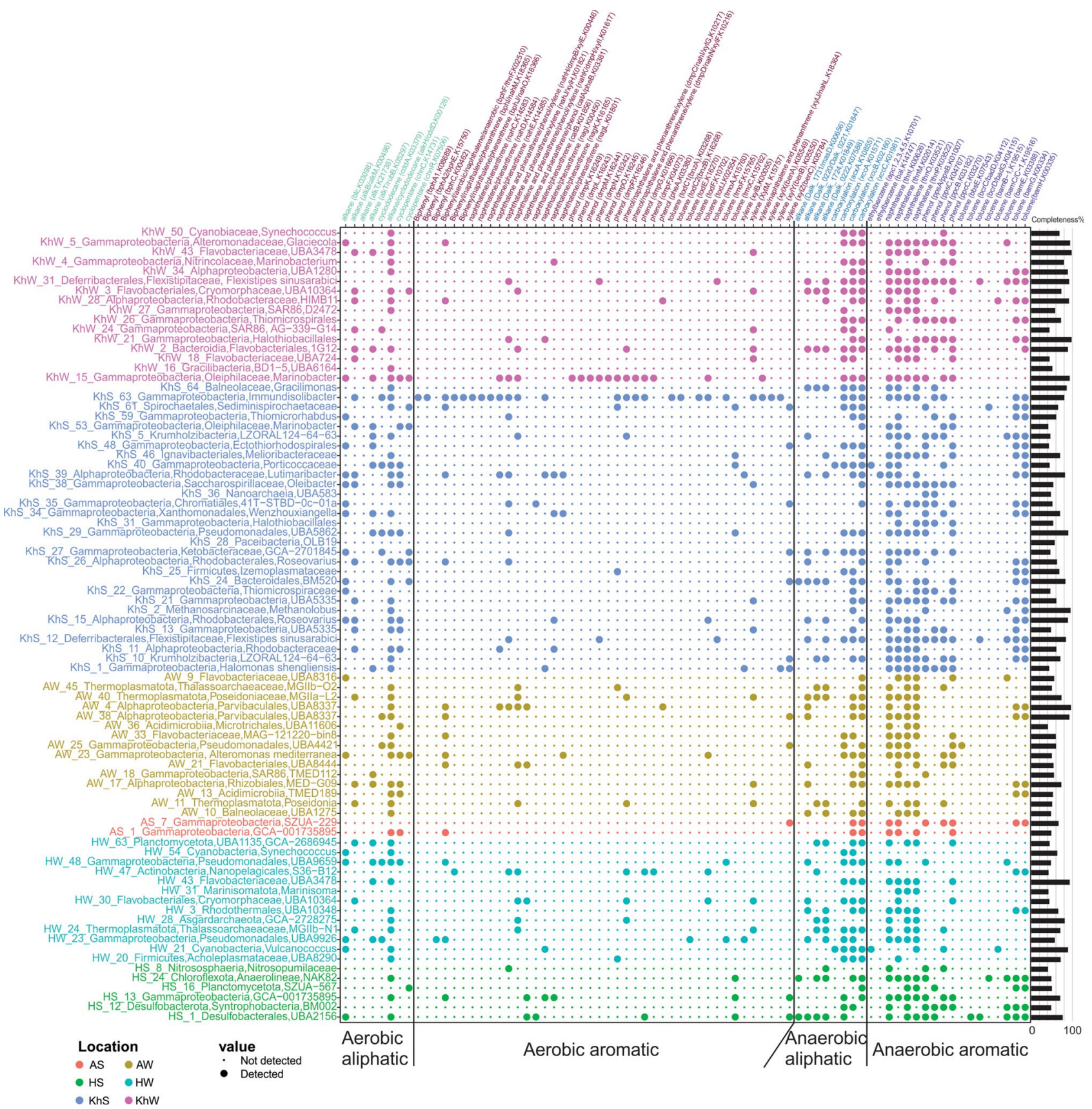

Figure 6. Hydrocarbon degrading enzymes present in recovered MAGs from the PG water and sediment metagenomes. Row names represent the taxonomy of recovered MAGs and their completeness is provided as a bar plot on the right side. The color indicates the MAG origin. The size of dots indicates the presence or absence of each enzyme in each recovered MAG. Columns indicate the type of hydrocarbon and in the parenthesis is the name of the enzyme hydrolyzing this compound followed by its corresponding KEGG orthologous accession number. Figure was plotted using "reshape2" and "ggplot2" packages in R.

degradation is mainly started by the addition of fumarate or in some cases, by carboxylation of the substrate. Therefore, bacteria containing these genes will potentially initiate the degradation process that will be continued by other heterotrophs. Enzymes such as decarboxylase, hydroxylase, dehydrogenase, hydratase, and isomerases act on the products of initiating enzymes mentioned above through a series of oxidation/reduction reactions.

Various microorganisms cooperate to cleave HCs into simpler compounds that could enter common metabolic pathways. Mono- or dioxygenases which are involved in the degradation of alkane (alkane 1-monooxygenase, alkB/alkM), cyclododecane (cyclododecanone monooxygenase, cddA), Biphenyl (Biphenyl 2, 3-dioxygenase subunit alpha/beta, bphA1/A2, Biphenyl-2, 3-diol 1, 2-dioxygenase, bphC), phenol (phenol 2-monooxygenase, pheA), toluene (benzene 1, 2-dioxygenase subunit alpha/beta todC1/C2, hydroxylase component of toluene4-monooxygenase, todE), xylene (toluate/benzoate 1,2-dioxygenase subunit alpha/beta/electron transport component, xylX/Y/Z, hydroxylase component of xylene monooxygenase, xylM) and naphthalene/phenanthrene 
(catechol 1,2 dioxygenase, catA, a shared enzyme between naphthalene/phenanthrene /phenol degradation) were detected in recovered MAGs of the PG.

The key enzymes including Alkylsuccinate synthase (I)/(II) (assA1/A2), benzylsuccinate synthase (BssA)/ benzoyl-CoA reductase (BcrA), ethylbenzene dehydrogenase (EbdA), and 6-oxo-cyclohex-1-ene-carbonyl-CoA hydrolase (BamA) that are responsible for initiating the degradation of alkane, toluene, ethylbenzene and benzoate exclusively under anaerobic conditions were not detected in reconstructed MAGs of this study. Consequently, recovered MAGs of this study are not initiating anaerobic degradation via known pathways while they have the necessary genes to continue the degradation process started by other microorganisms.

The MAG KhS_63 affiliated to Immundisolibacter contained various types of mono- or dioxygenases and had the potential to degrade a diverse range of HCs such as alkane, cyclododecane, toluene, and xylene (Fig. 6). Members of this genus have been reported to degrade high molecular weight $\mathrm{PAHs}^{51}$.

Lutimaribacter representatives have been isolated from seawater and reported to be capable of degrading cyclohexylacetate ${ }^{52}$. We also detected enzymes responsible for alkane, cycloalkane (even monooxygenase enzymes), and naphthalene degradation under aerobic conditions and alkane, ethylbenzene, toluene, and naphthalene degradation under anaerobic conditions in KhS_39 affiliated to this genus (Fig. 6).

The MAGs KhS_15 and KhS_26 affiliated to Roseovarius had the enzymes for degrading alkane (alkane monooxygenase, aldehyde dehydrogenase), cycloalkane, naphthalene, and phenanthrene under aerobic and toluene and naphthalene under anaerobic condition. PAHs degradation has been reported for other representatives of this taxa as well ${ }^{53}$.

The MAGs KhS_11 (a representative of Rhodobacteraceae) and KhS_53 (Marinobacter) had alkB/alkM, KhS_27 (GCA-2701845), KhS_29 (UBA5862) and KhS_40 (from Porticoccaceae family) had cddA, KhS_13 and KhS_21 (UBA5335) and KhS_38 (Oleibacter) had both alkB/alkM and xylM genes. They were among microbes that were initiating the degradation of alkane, cycloalkane and xylene compounds. Other MAGs recovered from Khark sediment were involved in the continuation of the degradation pathway. For example, KhS_1 was affiliated to the genus Halomonas and had different enzymes to degrade intermediate compounds. Halomonas representatives have been frequently isolated from oil-polluted environments $\mathrm{s}^{54}$. The phylum Krumholzibacteria has been first introduced in 2019 and reported to contain heterotrophic nitrite reducers ${ }^{55}$. Two MAGs, KhS_5 and KhS_10, were affiliated to this phylum and contained enzymes involved in the anaerobic degradation of toluene, phenol, and naphthalene (Fig. 6).

The MAGs KhS_12 and KhW_31 affiliated to the genus Flexistipes, in Deferribacterales order, were reconstructed from both KhW and KhS samples. Deferribacterales are reported to be present in the medium to hightemperature oil reservoirs with HC degradation activity and also in high-temperature oil-degrading consortia ${ }^{56}$. The type strain of this species was isolated from environments with a minimum salinity of $3 \%$ and a temperature of $45-50{ }^{\circ} \mathrm{C}^{57}$. The presence of this genus in $\mathrm{KhS}$ could be due to natural oil seepage from the seabed as PG reservoirs mainly have medium to high temperature and high salinity. Enzymes involved in the degradation of alkane, phenol, toluene and naphthalene under anaerobic conditions were present in MAGs KhS_12 and KhW_31.

As mentioned earlier, Flavobacteriales are potent marine indigenous $\mathrm{HC}$ degraders that bloom in response to oil pollution ${ }^{58}$. Flavobacteriales affiliated MAGs (KhW_2, KhW_3, AW_21, and AW_33) were recovered from KhW and AW and mostly contained enzymes that participate in the degradation of aromatic compounds under anaerobic conditions. KhW_2 and KhW_3 also had both alkB/M (alkane monooxygenase) and xylM enzyme, which initiates the alkane and xylene bioremediation in Khark water. Among other recovered MAGs from KhW sample, KhW_18 (UBA724), KhW_24 (clade SAR86), KhW_43 (UBA3478) had alkB/M, and xylM, KhW_24 (clade SAR86) had alkB/M and cdd $\bar{A}$, and $\mathrm{KhW} 28$ (from Rhodobacteraceae family) had alkB/M and pheA genes in their genome to initiate the degradation process (Fig. 6).

Marinobacter (KhW_15) was another MAG reconstructed from KhW sample. This genus is one of the main cultivable genera that play a crucial role in the bioremediation of a wide range of oil derivatives in polluted marine ecosystems ${ }^{54}$.

Marine Group II (MGII) and Poseidonia representatives of Thermoplasmatota that have been reported to be nitrate-reducing Archaea ${ }^{59}$, were recovered from AW sample (AW_40, AW_45) and contained several enzymes contributing in alkane (alkane monooxygenase, aldehyde dehydrogenase) and naphthalene/phenanthrene/phenol/xylene degradation (decarboxylase) under aerobic conditions. The HC degradation potential of representatives of this phylum has been previously reported ${ }^{60}$.

In the Asalouyeh water sample, MAGs AW_25 (UBA4421) and AW_38 (UBA8337) had cddA, AW_21 (UBA8444) had catA, AW_11 (Poseidonia) and AW_17 (from Rhizobiales order) had both alkB/M and xylM, and AW_4 (UBA8337) had catA and pheA genes and had potential to trigger the breakdown of their corresponding oil derivatives.

Other recovered genomes had the potential to metabolize the product of initiating enzymes. For instance, AW_23 contained enzymes involved in the degradation of naphthalene, phenol and cyclododecane and was affiliated to the genus Alteromonas (Fig. 6).

Three recovered MAGs of HW affiliated to Pseudomonadales (HW_23), Poseidoniales (HW_24), and Flavobacteriales (HW_30) contained some initiating enzymes to degrade cyclododecane/biphenyl/toluene, alkane/ xylene, and alkane/xylene/naphthalene/phenanthrene, respectively. A representative of Heimdallarchaeia that are mainly recovered from sediment samples was reconstructed from the Hormuz water sample (HW_28). It had a completeness of $81 \%$ and contained enzymes involved in anaerobic degradation of alkanes. This archaeon could potentially be an input from the neighboring OMZ as this phyla include representatives adopted to microoxic niches ${ }^{61}$. Containing genes with the potential to initiate the oil derivative degradation in the input water with no oil exposure reiterates the intrinsic ability of marine microbiota for HC degradations and oil bioremediation.

While $16 \mathrm{~S}$ rRNA provides an overview of the community, MAGs provide the possibility to inspect the metabolic capability of the microbiota. We decided to provide both in this manuscript as we believe they are 

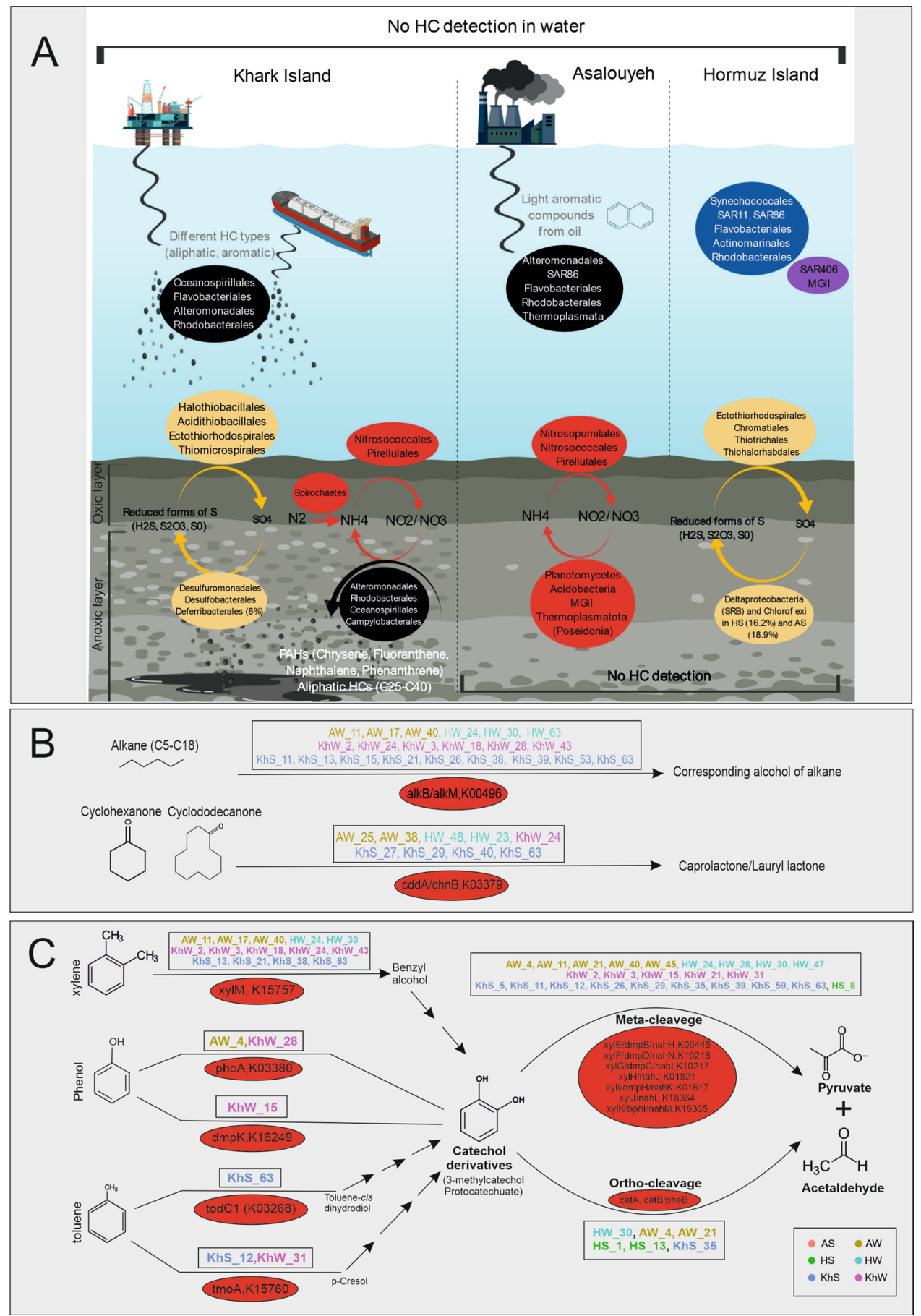

Figure 7. The microbial community dynamics of the Persian Gulf water and sediment samples in response to oil pollution and their degradation potential. (A) Overview based on $16 \mathrm{~S}$ rDNA abundance. Hormuz Island was considered as a control location with the least impact from oil pollution. Taxa written in the blue frame are prevalent marine representatives present in HW. Microbial taxa in the Purple frame are mainly detected in OMZ areas and are also present in HW. Samples collected from Asalouyeh province are exposed to potential pollution caused by Gas field wastes. High oil trafficking, oil exploration and extraction, and natural oil seepage are the primary potential pollution sources in Khark Island. The possible pollutant types are shown in gray; however, the hydrocarbon pollution was below the detection limit in collected water samples. Black circles represent microorganisms that are involved in HC degradation in water samples from Asalouyeh and Khark Island. Microbes involved in sulfur and nitrogen cycle are shown in yellow and Red circles, respectively. HS and AS had similar silt and sand-sized sediments with HC below the detection limit. KhS had gravel-sized particles and showed the highest oil pollution shown in white. (B) MAGs containing key enzymes for degradation of aliphatic and cycloalkane compounds under aerobic conditions. (C) MAGs containing key enzymes for degradation of aromatic compounds under aerobic conditions. PG MAGs did not have Key enzymes for hydrocarbon degradation under anaerobic conditions therefore, it is not shown. Red circles in B and C panels represent key enzymes involved in the degradation. The name of MAGs containing mentioned enzymes are written in rectangles. The MAGs are colored based on the samples they have been recovered from and the legend is shown in the lower right corner. Figure has been created with "BioRender.com". 
complementary. Having the full picture provided by the combination of these analyses allows for a better understanding of the community structure and their metabolic capabilities. This is even more evident for sediment samples as they are highly diverse, and reconstructing MAGs from sediment metagenomes is still a bottlenecks. In this case, we rely more on the $16 \mathrm{~S}$ rRNA to provide an overall view of the community composition.

This said, we see similar taxonomic distribution in the MAGs and 16S rRNA e.g., the prevalence of Flavobacteriales and Rhodobacterales in KhW and KhS, Synechococcales, and Desulfobacteriales and Flavobacteriales in HW, HS and AW samples, respectively.

Additionally, some rare microbiota representatives were recovered among reconstructed MAGs. For example, the Immundisolibacterales showed an abundance of only $0.8 \%$ in the KhS sample based on $16 \mathrm{~S}$ rRA but the recovered KhS_63 MAG was affiliated to this taxon. Notably, this MAG contained many genes involved in hydrocarbon degradation having the highest potential in hydrocarbon degradation.

\section{Conclusion}

Exploring the marine microbial communities' response to oil pollutions has received increasing attention over the last decade, specifically after the "Deepwater Horizon oil spill". However, the influence of long-term exposure to oil derivatives in ecosystems such as the Persian Gulf that hosts almost half of the world's oil reserves and has been chronically exposed to recurrent natural and accidental oil pollutions has remained entirely unknown. Understanding the microbial dynamics in response to oil pollution at different locations of the Persian Gulf can function as a valuable model system for advancing our knowledge and preparedness for managing oil spill accidents in the future.

Our extensive analysis of available oil-polluted water and sediment metagenomes $(n=106)$, together with the Persian Gulf samples $(n=6)$, showed that the chronic exposure to trace amounts of oil derivatives had altered the microbial community of the Persian Gulf. Even though the pollution remained below our detection limit of $50 \mu \mathrm{g} / \mathrm{L}$, the long-standing trace oil pollution imposed a consistent selection pressure on the microbial community of the input water, selecting for oil-degrading microbes capable of degrading major locally enriched pollutants (Fig. 7).

Our results showed that regardless of the water column depth and initial composition, the microbial community converges in response to the pollution. It seems that microbes capable of degrading more labile components of the pollutant will be recruited to the pollution zone. Their population will experience a bloom, which will be followed by the next populations capable of degrading more recalcitrant components. Our genome-resolved analyses showed that these microbes employ an intricate "division of labor" to initiate and carry out different stages of the bioremediation process. Higher-resolution spatiotemporal analysis of the microbial community of this highly heterogeneous ecosystem in future studies can reveal critical ecological adaptations to oil pollutants.

\section{Data availability}

The metagenomic Raw read files of the Persian Gulf water and sediment samples and all the metagenomeassembled genomes (MAGs) reconstructed (Supplementary Table S5) in this study are archived at the DDBJ/ EMBL/GenBank and can be accessed under the Bioproject PRJNA575141.

Received: 29 October 2020; Accepted: 17 May 2021

Published online: 31 May 2021

\section{References}

1. Brussaard, C. P. D. et al. Immediate ecotoxicological effects of short-lived oil spills on marine biota. Nat. Commun. 7, 11206 (2016).

2. Joydas, T. V., Qurban, M. A., Borja, A., Krishnakumar, P. K. \& Al-Suwailem, A. Macrobenthic community structure in the Northwestern Arabian Gulf, twelve years after the 1991 oil spill. Front. Mar. Sci. 4, 248 (2017).

3. Pous, S., Lazure, P. \& Carton, X. A model of the general circulation in the Persian Gulf and in the Strait of Hormuz: Intraseasonal to interannual variability. Cont. Shelf Res. 94, 55-70 (2015).

4. Yergeau, E. et al. Microbial community composition, functions, and activities in the Gulf of Mexico 1 year after the deepwater horizon accident. Appl. Environ. Microbiol. 81, 5855-5866 (2015).

5. Hu, P. et al. Simulation of Deepwater Horizon oil plume reveals substrate specialization within a complex community of hydrocarbon degraders. Proc. Natl. Acad. Sci. 114, 7432-7437 (2017).

6. Ludt, W. B., Morgan, L., Bishop, J. \& Chakrabarty, P. A quantitative and statistical biological comparison of three semi-enclosed seas: the Red Sea, the Persian (Arabian) Gulf, and the Gulf of California. Mar. Biodivers. 48, 2119-2124 (2018).

7. Hassanshahian, M. \& Boroujeni, N. A. Enrichment and identification of naphthalene-degrading bacteria from the Persian Gulf. Mar. Pollut. Bull. 107, 59-65 (2016).

8. Hassanshahian, M., Emtiazi, G. \& Cappello, S. Isolation and characterization of crude-oil-degrading bacteria from the Persian Gulf and the Caspian Sea. Mar. Pollut. Bull. 64, 7-12 (2012).

9. Ghanbari, M., Jami, M., Moghadam, M. S. \& Domig, K. J. Exploring the microbial diversity in oil-contaminated mangrove sediments using 16S rRNA metagenomics. bioRxiv 1, 735290 (2019).

10. Al Azhar, M., Temimi, M., Zhao, J. \& Ghedira, H. Modeling of circulation in the Arabian Gulf and the Sea of Oman: Skill assessment and seasonal thermohaline structure. J. Geophys. Res. Ocean. 121, 1700-1720 (2016).

11. Delshab, H., Farshchi, P., Mohammadi, M. \& Moattar, F. Preliminary assessment of heavy metal contamination in water and wastewater from Asaluyeh Port (Persian Gulf). Iran. J. Sci. Technol. Trans. A Sci. https://doi.org/10.1007/s40995-016-0031-x (2016).

12. Akhbarizadeh, R., Moore, F., Keshavarzi, B. \& Moeinpour, A. Aliphatic and polycyclic aromatic hydrocarbons risk assessment in coastal water and sediments of Khark Island, SW Iran. Mar. Pollut. Bull. 108, 33-45 (2016).

13. Segev, E. et al. Dynamic metabolic exchange governs a marine algal-bacterial interaction. Elife 5, e17473 (2016).

14. Adeniji, A. O., Okoh, O. O. \& Okoh, A. I. Analytical methods for the determination of the distribution of total petroleum hydrocarbons in the water and sediment of aquatic systems: A review. J. Chem. 2017, 1-13 (2017).

15. Poster, D. L., Schantz, M. M., Sander, L. C. \& Wise, S. A. Analysis of polycyclic aromatic hydrocarbons (PAHs) in environmental samples: A critical review of gas chromatographic (GC) methods. Anal. Bioanal. Chem. 386, 859-881 (2006).

16. Boczkaj, G., Przyjazny, A. \& Kamiński, M. A new procedure for the determination of distillation temperature distribution of highboiling petroleum products and fractions. Anal. Bioanal. Chem. 399, 3253-3260 (2011). 
17. Martín-Cuadrado, A.-B. et al. Metagenomics of the deep Mediterranean, a warm bathypelagic habitat. PLoS One 2, e914 (2007).

18. Marmur, J. A procedure for the isolation of deoxyribonucleic acid from micro-organisms. J. Mol. Biol. 3, 208 (1961).

19. Jiang, H. et al. Microbial diversity in water and sediment of Lake Chaka, an athalassohaline lake in northwestern China. Appl. Environ. Microbiol. 72, 3832-3845 (2006).

20. Mori, H. et al. Design and experimental application of a novel non-degenerate universal primer set that amplifies prokaryotic $16 \mathrm{~S}$ rRNA genes with a low possibility to amplify eukaryotic rRNA genes. DNA Res. 21, 217-227 (2013).

21. Naghoni, A. et al. Microbial diversity in the hypersaline Lake Meyghan, Iran. Sci. Rep. 7, 11522 (2017).

22. Nawrocki, E. P. Structural RNA Homology Search and Alignment Using Covariance Models. PhD thesis, Washington University School of Medicine (2009).

23. Bushnell, B. BBMap: A Fast, Accurate, Splice-Aware Aligner. Lawrence Berkeley National Laboratory LBNL Report \#: LBNL-7065E. (2014). Retrieved from https://escholarship.org/uc/item/1h3515gn

24. Li, D., Liu, C.-M., Luo, R., Sadakane, K. \& Lam, T.-W. MEGAHIT: An ultra-fast single-node solution for large and complex metagenomics assembly via succinct de Bruijn graph. Bioinformatics 31, 1674-1676 (2015).

25. Kang, D. et al. MetaBAT 2: An adaptive binning algorithm for robust and efficient genome reconstruction from metagenome assemblies. PeerJ Prepr. 7, e27522v1 (2019).

26. Parks, D. H., Imelfort, M., Skennerton, C. T., Hugenholtz, P. \& Tyson, G. W. CheckM: Assessing the quality of microbial genomes recovered from isolates, single cells, and metagenomes. Genome Res. 25, 1043-1055 (2015).

27. Parks, D. H. et al. A standardized bacterial taxonomy based on genome phylogeny substantially revises the tree of life. Nat. Biotechnol. 36, 996-1004 (2018).

28. Hyatt, D. et al. Prodigal: Prokaryotic gene recognition and translation initiation site identification. BMC Bioinform. 11, 119 (2010).

29. Seemann, T. Prokka: Rapid prokaryotic genome annotation. Bioinformatics 30, 2068-2069 (2014).

30. Huerta-Cepas, J. et al. eggNOG 5.0: A hierarchical, functionally and phylogenetically annotated orthology resource based on 5090 organisms and 2502 viruses. Nucleic Acids Res. 47, D309-D314 (2018).

31. Finn, R. D. et al. The Pfam protein families database: Towards a more sustainable future. Nucleic Acids Res. 44, D279-D285 (2016).

32. Fuhrman, J. A., Cram, J. A. \& Needham, D. M. Marine microbial community dynamics and their ecological interpretation. Nat. Rev. Microbiol. 13, 133-146 (2015).

33. Treusch, A. H. et al. Seasonality and vertical structure of microbial communities in an ocean gyre. ISME J. 3, 1148-1163 (2009).

34. Bacosa, H. P., Liu, Z. \& Erdner, D. L. Natural sunlight shapes crude oil-degrading bacterial communities in Northern Gulf of Mexico surface waters. Front. Microbiol. 6, 1325 (2015).

35. King, G. M., Kostka, J. E., Hazen, T. C. \& Sobecky, P. A. Microbial responses to the Deepwater Horizon oil spill: From coastal wetlands to the deep sea. Ann. Rev. Mar. Sci. 7, 377-401 (2015).

36. Dupont, C. L. et al. Genomic insights to SAR86, an abundant and uncultivated marine bacterial lineage. ISME J. 6, 1186-1199 (2012).

37. Mason, O. U. et al. Metagenomics reveals sediment microbial community response to Deepwater Horizon oil spill. ISME J. 8, 1464 (2014).

38. Liu, Y.-F. et al. Metabolic capability and in situ activity of microorganisms in an oil reservoir. Microbiome 6, 5 (2018).

39. de Sousa, L. S., Wambua, R. M., Raude, J. M. \& Mutua, B. M. Assessment of water flow and sedimentation processes in irrigation schemes for decision-support tool development: A case review for the Chókwè irrigation scheme, Mozambique. AgriEngineering 1, 100-118 (2019).

40. Gutierrez T. (2019) Marine, Aerobic Hydrocarbon-Degrading Gammaproteobacteria: Overview. In: McGenity T. (eds) Taxonomy, Genomics and Ecophysiology of Hydrocarbon-Degrading Microbes. Handbook of Hydrocarbon and Lipid Microbiology. Springer, Cham.

41. Bertagnolli, A. D. \& Stewart, F. J. Microbial niches in marine oxygen minimum zones. Nat. Rev. Microbiol. 16, 723-729 (2018).

42. Thrash, J. C. et al. Metabolic roles of uncultivated bacterioplankton lineages in the Northern Gulf of Mexico 'dead zone'. MBio 8, e01017-e1117 (2017)

43. Abbasian, F., Lockington, R., Megharaj, M. \& Naidu, R. A review on the genetics of aliphatic and aromatic hydrocarbon degradation. Appl. Biochem. Biotechnol. 178, 224-250 (2016).

44. Stagars, M. H., Mishra, S., Treude, T., Amann, R. \& Knittel, K. Microbial community response to simulated petroleum seepage in Caspian Sea sediments. Front. Microbiol. 8, 764 (2017).

45. Davidova I. A., Marks C. R., Suflita J. M. (2019) Anaerobic Hydrocarbon-Degrading Deltaproteobacteria In: McGenity T. (eds) Taxonomy, Genomics and Ecophysiology of Hydrocarbon-Degrading Microbes. Handbook of Hydrocarbon and Lipid Microbiology. Springer, Cham.

46. Shin, B., Bociu, I., Kolton, M., Huettel, M. \& Kostka, J. E. Succession of microbial populations and nitrogen-fixation associated with the biodegradation of sediment-oil-agglomerates buried in a Florida sandy beach. Sci. Rep. 9, 1-11 (2019).

47. Acosta-González, A., Rosselló-Móra, R. \& Marqués, S. Characterization of the anaerobic microbial community in oil-polluted subtidal sediments: Aromatic biodegradation potential after the Prestige oil spill. Environ. Microbiol. 15, 77-92 (2013).

48. Abbasian, F., Lockington, R., Mallavarapu, M. \& Naidu, R. A comprehensive review of aliphatic hydrocarbon biodegradation by bacteria. Appl. Biochem. Biotechnol. 176, 670-699 (2015).

49. Rabus, R. et al. Anaerobic microbial degradation of hydrocarbons: From enzymatic reactions to the environment. J. Mol. Microbiol. Biotechnol. 26, 5-28 (2016).

50. Espínola, F. et al. Metagenomic analysis of subtidal sediments from polar and subpolar coastal environments highlights the relevance of anaerobic hydrocarbon degradation processes. Microb. Ecol. 75, 123-139 (2018).

51. Corteselli, E. M., Aitken, M. D. \& Singleton, D. R. Description of Immundisolibacter cernigliae gen. nov., sp. nov., a high-molecularweight polycyclic aromatic hydrocarbon-degrading bacterium within the class Gammaproteobacteria, and proposal of Immundisolibacterales ord. nov. and Immundisolibacteraceae f. Int. J. Syst. Evol. Microbiol. 67, 925 (2017).

52. Iwaki, H., Yasukawa, N., Fujioka, M., Takada, K. \& Hasegawa, Y. Isolation and characterization of a marine cyclohexylacetatedegrading bacterium Lutimaribacter litoralis sp. nov., and reclassification of Oceanicola pacificus as Lutimaribacter pacificus comb. nov.. Curr. Microbiol. 66, 588-593 (2013).

53. Shao, Z., Yuan, J., Lai, Q. \& Zheng, T. The diversity of PAH-degrading bacteria in a deep-sea water column above the Southwest Indian Ridge. Front. Microbiol. 6, 853 (2015)

54. Barbato, M., Mapelli, F., Crotti, E., Daffonchio, D. \& Borin, S. Cultivable hydrocarbon degrading bacteria have low phylogenetic diversity but highly versatile functional potential. Int. Biodeterior. Biodegrad. 142, 43-51 (2019).

55. Youssef, N. H. et al. Candidatus Krumholzibacterium zodletonense gen. nov., sp nov., the first representative of the candidate phylum Krumholzibacteriota phyl. nov. recovered from an anoxic sulfidic spring using genome resolved metagenomics. Syst. Appl. Microbiol. 42, 85-93 (2019).

56. Zhou, Z., Liang, B., Wang, L.-Y., Liu, J.-F., Mu, B.-Z., Shim, H., \& Gu, J.-D. Identifying the core bacterial microbiome of hydrocarbon degradation and a shift of dominant methanogenesis pathways in the oil and aqueous phases of petroleum reservoirs of different temperatures from China. Biogeosciences 16, 4229-4241, 2019.

57. Fiala, G., Woese, C. R., Langworthy, T. A. \& Stetter, K. O. Flexistipes sinusarabici, a novel genus and species of eubacteria occurring in the Atlantis II Deep brines of the Red Sea. Arch. Microbiol. 154, 120-126 (1990). 
58. Liu, Z. \& Liu, J. Evaluating bacterial community structures in oil collected from the sea surface and sediment in the northern Gulf of Mexico after the Deepwater Horizon oil spill. Microbiologyopen 2, 492-504 (2013).

59. Rinke, C., Rubino, F., Messer, L.F. et al. A phylogenomic and ecological analysis of the globally abundant Marine Group II archaea (Ca. Poseidoniales ord. nov.). ISME J 13, 663-675 (2019).

60. Jeanbille, M. et al. Response of core microbial consortia to chronic hydrocarbon contaminations in coastal sediment habitats. Front. Microbiol. 7, 1637 (2016).

61. Bulzu, P.-A. et al. Casting light on Asgardarchaeota metabolism in a sunlit microoxic niche. Nat. Microbiol. 4, 1129-1137 (2019).

\title{
Acknowledgements
}

This study was supported by a grant of young pioneer biotechnologists from Iran Council for the development of Biotechnology to SMMD and the Iranian National Science Foundation (INSF) (to MAA). M.M. acknowledges the Science for Life Laboratory. The computational analysis was performed at the Center for High-Performance Computing, School of Mathematics, Statistics, and Computer Science, University of Tehran.

\section{Author contributions}

M.A.A., S.M.M.D., M.M., and M.S. devised the study. M.R.S. and S.M.M.D. collected and processed the samples. M.R.S., M.M., and L.G.M. performed the bioinformatics analysis with assistance from K.K. M.R.S. and M.M. drafted the manuscript. All authors read and approved the manuscript.

\section{Funding}

Open access funding provided by Uppsala University.

\section{Competing interests}

The authors declare no competing interests.

\section{Additional information}

Supplementary Information The online version contains supplementary material available at https://doi.org/ 10.1038/s41598-021-90735-0.

Correspondence and requests for materials should be addressed to M.A.A. or M.M.

Reprints and permissions information is available at www.nature.com/reprints.

Publisher's note Springer Nature remains neutral with regard to jurisdictional claims in published maps and institutional affiliations.

\begin{abstract}
(c) (i)
Open Access This article is licensed under a Creative Commons Attribution 4.0 International License, which permits use, sharing, adaptation, distribution and reproduction in any medium or format, as long as you give appropriate credit to the original author(s) and the source, provide a link to the Creative Commons licence, and indicate if changes were made. The images or other third party material in this article are included in the article's Creative Commons licence, unless indicated otherwise in a credit line to the material. If material is not included in the article's Creative Commons licence and your intended use is not permitted by statutory regulation or exceeds the permitted use, you will need to obtain permission directly from the copyright holder. To view a copy of this licence, visit http://creativecommons.org/licenses/by/4.0/.
\end{abstract}

(C) The Author(s) 2021 